Homology, Homotopy and Applications, vol.17(1), 2015, pp.129-146

\title{
OPERATIONS ON THE SECONDARY HOCHSCHILD COHOMOLOGY
}

\author{
MIHAI D. STAIC AND ALIN STANCU \\ (communicated by Nathalie Wahl)
}

\begin{abstract}
We show that the secondary Hochschild cohomology associated to a triple $(A, B, \varepsilon)$ has several of the properties of the usual Hochschild cohomology. Among others, we prove that the total secondary Hochschild complex is a multiplicative nonsymmetric operad, discuss the connection with extensions of $B$-algebras, and give a Hodge-type decomposition of the secondary Hochschild cohomology.
\end{abstract}

In memory of our advisor, Dr. Samuel D. Schack

\section{Introduction}

Hochschild cohomology was introduced by Hochschild in [8] in order to study extensions of associative algebras over a field and to characterize the separability of this class of algebras. In the same paper (written while he was a draftee serving in the army) he defined for any associative algebra $A$ the cup product of cochains with coefficients in $A$. From Hochschild's definition it follows easily that the cup product on the cochains descends to one on the Hochschild cohomology $H^{\bullet}(A, A)$. Almost twenty years later Gerstenhaber proved in $[\mathbf{3}]$ that at the cohomology level the cup product is graded commutative. He also defined a Lie product whose properties, when combined with those of the cup product, determine on $H^{\bullet}(A, A)$ a rich algebraic structure which is now called a Gerstenhaber algebra (or $G$-algebra). $G$-algebra structures appear in other contexts of which we mention here the exterior algebra of a Lie algebra, the differential forms on a Poisson manifold, and the Hochschild cohomology of presheaves of algebras. Part of this paper is to show that the secondary Hochschild cohomology can be added to this list.

Consider a $B$-algebra $A$ determined by the $k$-algebra homomorphism $\varepsilon: B \rightarrow A$. The secondary Hochschild cohomology of the triple $(A, B, \varepsilon)$ with coefficients in $A$, denoted $H^{\bullet}((A, B, \varepsilon) ; A)$, arises naturally in the study of the algebra deformations $A[[t]]$ which admit a $B$-algebra structure. It was introduced in $[\mathbf{1 2}]$ where it was proved that a $B$-algebra structure on $A[[t]]$ is determined by a family of products

The first author was partially supported by a grant of the Romanian National Authority for Scientific Research, CNCS-UEFISCDI, project number PN-II-ID-PCE-2011-3-0635, contract 253/5.10.2011. Received May 30, 2014, revised October 28, 2014; published on April 13, 2015. 2010 Mathematics Subject Classification: Primary 16E40, Secondary 16S80.

Key words and phrases: Hochschild cohomology, deformation of algebras. Article available at http://dx.doi.org/10.4310/HHA.2015.v17.n1.a6

Copyright (C) 2015, International Press. Permission to copy for private use granted. 
$m_{\alpha}: A[[t]] \otimes A[[t]] \rightarrow A[[t]]$ that must satisfy the following generalized associativity condition: $m_{\alpha \beta}\left(i d \otimes m_{\gamma}\right)=m_{\beta \gamma}\left(m_{\alpha} \otimes i d\right)$ for all $\alpha, \beta$ and $\gamma \in B$. For $a, b \in A$ and $\alpha \in B$ we have $m_{\alpha}(a \otimes b)=\varepsilon(\alpha) a b+c_{1}(a \otimes b \otimes \alpha) t+\cdots$. Just like in the case of deformations of algebras, $c_{1}$ is a 2-cocycle that gives the deformation $\bmod t^{2}$. Its class $c_{1} \in H^{2}((A, B, \varepsilon) ; A)$ is determined by the isomorphism class of the $B$-algebra $A[[t]]$. Moreover, if we assume that $m_{\alpha}$ is associative $\bmod t^{n+1}$ then the obstruction to extend it to an associative product $\bmod t^{n+2}$ is the vanishing of the element $c_{1}$ 。 $c_{n}+c_{2} \circ c_{n-1}+\cdots+c_{n} \circ c_{1}$ in $H^{3}((A, B, \varepsilon) ; A)$.

The paper is organized as follows. In the first section we recall the definition of the secondary Hochschild cohomology and some results about operads. In the second we show that the secondary Hochschild complex is a multiplicative non-symmetric operad and that the secondary Hochschild cohomology has a $G$-algebra structure. In the third section we show that there is bijection between extensions of $B$-algebras $0 \rightarrow M \rightarrow X \rightarrow A \rightarrow 0$ with $M^{2}=0$ and $H^{2}((A, B, \varepsilon) ; M)$. In the fourth we give a Hodge-type decomposition, in characteristic 0 , for the secondary cohomology, one that is consistent with the Hodge decomposition of the usual Hochschild cohomology. Finally, in the last section we investigate the (cup and bracket preserving) natural map $\Phi: H^{n}((A, B, \varepsilon) ; A) \rightarrow H^{n}(A, A)$. More precisely, we present examples which show that in general $\Phi$ is neither surjective nor injective. Our examples deal with subalgebras of the ring of polynomials. We show that requiring $\Phi_{2}$ to be injective is equivalent to the Jacobian problem stated in [13], a question first posed by OttHeinrich Keller in 1939.

\section{Preliminaries}

\subsection{Hochschild cohomology of an algebra $A$}

In this paper $k$ is a field, $\otimes=\otimes_{k}$, and all $k$-algebras have a multiplicative unit. We recall from [4] and [9] the definition of the Hochschild cohomology.

Suppose that $A$ is an associative $k$-algebra, and $M$ is an $A$-bimodule. Define $C^{n}(A, M)=H_{o m}\left(A^{\otimes n}, M\right)$ and $\delta_{n}: C^{n}(A, M) \rightarrow C^{n+1}(A, M)$ determined by:

$$
\begin{aligned}
\delta_{n}(f)\left(a_{1} \otimes a_{2} \otimes \cdots \otimes a_{n+1}\right)= & a_{1} f\left(a_{2} \otimes \cdots \otimes a_{n+1}\right) \\
& +\sum_{i=1}^{n}(-1)^{i} f\left(a_{1} \otimes \cdots \otimes a_{i} a_{i+1} \otimes \cdots \otimes a_{n+1}\right) \\
& +(-1)^{n+1} f\left(a_{1} \otimes \cdots \otimes a_{n}\right) a_{n+1} .
\end{aligned}
$$

One can show that $\delta_{n+1} \delta_{n}=0$. The homology of this complex is denoted by $H^{n}(A, M)$ and is called the Hochschild cohomology of $A$ with coefficients in $M$.

\subsection{Secondary cohomology of a triple $(A, B, \varepsilon)$}

We recall from $[\mathbf{1 2}]$ the definition of the secondary Hochschild cohomology.

Let $A$ be an associative $k$-algebra; $B$ a commutative $k$-algebra; $\varepsilon: B \rightarrow A$ a morphism of $k$-algebras such that $\varepsilon(B) \subset \mathcal{Z}(A)$, the center of $A$; and $M$ an $A$-bimodule. We assume that for every $\alpha \in B$ and $m \in M$ we have $\varepsilon(\alpha) m=m \varepsilon(\alpha)$. Let

$$
C^{n}((A, B, \varepsilon) ; M)=H_{o m}\left(A^{\otimes n} \otimes B^{\otimes \frac{n(n-1)}{2}}, M\right) .
$$

We want to define $\delta_{n}^{\varepsilon}: C^{n}((A, B, \varepsilon) ; M) \rightarrow C^{n+1}((A, B, \varepsilon) ; M)$. 
It is convenient to think about an element $T \in A^{\otimes n} \otimes B^{\otimes \frac{n(n-1)}{2}}$ using the following matrix representation:

$$
T=\otimes\left(\begin{array}{cccccc}
a_{1} & b_{1,2} & \cdots & b_{1, n-2} & b_{1, n-1} & b_{1, n} \\
1 & a_{2} & \cdots & b_{2, n-2} & b_{2, n-1} & b_{2, n} \\
\vdots & \vdots & & \vdots & \vdots & \vdots \\
1 & 1 & \cdots & 1 & a_{n-1} & b_{n-1, n} \\
1 & 1 & \cdots & 1 & 1 & a_{n}
\end{array}\right)
$$

where $a_{i} \in A, b_{i, j} \in B$, and $1 \in k$. Notice that we do not have exactly the same notation as in [12]; the difference here is that all the indices are shifted by one.

For $T \in A^{\otimes m+n-1} \otimes B^{\otimes \frac{(m+n-1)(m+n-2)}{2}}$ and for all $0 \leqslant i \leqslant m-1$ we denote by $T_{i+n}^{i}$ the following "sub-tensor matrix":

$$
T_{i+n}^{i}=\otimes\left(\begin{array}{ccc}
a_{i+1} & \cdots & b_{i+1, i+n} \\
\vdots & & \vdots \\
1 & \cdots & a_{i+n}
\end{array}\right) .
$$

One should notice that unless $i=0$ it does not make sense to talk about $T_{i+n}^{i}$ as a tensor but only as a sub-tensor of $T$. Clearly we have $T=T_{n}^{0}$.

For a tensor matrix $T \in A^{\otimes n} \otimes B^{\otimes \frac{n(n-1)}{2}}$ and positive integers $l, i$, and $k$ such that $1 \leqslant l \leqslant i \leqslant k \leqslant n-1$ we consider the sub-tensor matrix

$$
M_{i, i+1}^{l, k}=\otimes\left(\begin{array}{ccccccc}
a_{l} & b_{l, 2} & \cdots & b_{l, i} b_{l, i+1} & \cdots & b_{l, k} & b_{l, k+1} \\
1 & a_{l+1} & \cdots & b_{l+1, i} b_{l+1, i+1} & \cdots & b_{l+1, k} & b_{l+1, k+1} \\
\vdots & \vdots & & \vdots & & \vdots & \vdots \\
1 & 1 & \cdots & \varepsilon\left(b_{i, i+1}\right) a_{i} a_{i+1} & \cdots & b_{i, k} b_{i+1, k} & b_{i, k+1} b_{i+1, k+1} \\
\vdots & \vdots & & \vdots & & \vdots & \vdots \\
1 & 1 & \cdots & & \cdots & a_{k} & b_{k, k+1} \\
1 & 1 & \cdots & & \cdots & 1 & a_{k+1}
\end{array}\right) .
$$

With the above notation we define

$$
\begin{gathered}
\delta_{n}^{\varepsilon}: C^{n}((A, B, \varepsilon) ; M) \rightarrow C^{n+1}((A, B, \varepsilon) ; M), \\
\delta_{n}^{\varepsilon}(f)\left(T_{n+1}^{0}\right)= \\
a_{1} \varepsilon\left(b_{1,2} b_{1,3} \cdots b_{1, n+1}\right) f\left(T_{n+1}^{1}\right)-f\left(M_{1,2}^{1, n}\right)+f\left(M_{2,3}^{1, n}\right) \\
+\cdots+(-1)^{i} f\left(M_{i, i+1}^{1, n}\right)+\cdots+(-1)^{n-1} f\left(M_{n-1, n}^{1, n}\right)+(-1)^{n} f\left(M_{n, n+1}^{1, n}\right) \\
+(-1)^{n+1} f\left(T_{n}^{0}\right) a_{n+1} \varepsilon\left(b_{1, n+1} b_{2, n+1} \cdots b_{n, n+1}\right) .
\end{gathered}
$$

Proposition 2.1. ([12]) $\left(C^{n}((A, B, \varepsilon) ; M), \delta_{n}^{\varepsilon}\right)$ is a complex (i.e. $\left.\delta_{n+1}^{\varepsilon} \delta_{n}^{\varepsilon}=0\right)$. We denote its homology by $H^{n}((A, B, \varepsilon) ; M)$ and we call it the secondary Hochschild cohomology of the triple $(A, B, \varepsilon)$ with coefficients in $M$.

Example 2.2. When $B=k$ and $\varepsilon: k \rightarrow A$ is the map defining the $k$-algebra structure on $A$, we have that $H^{n}((A, k, \varepsilon) ; M)$ is the usual Hochschild cohomology. 


\subsection{Non-symmetric operads}

We recall from $[\mathbf{1}],[\mathbf{7}]$, and $[\mathbf{1 0}]$ a few results about non-symmetric operads. The general context in which operads are defined is that of a monoidal category $\mathcal{C}$ (see $[\mathbf{1 0}]$ ). We need these results for vector spaces over a field and we denote the multiplication on $\mathcal{C}$ by $\otimes$ and the unit object by 1 . For our purpose the most convenient definition of non-symmetric unital operads is the following:

Definition 2.3. A non-symmetric unital operad is a sequence of objects $\mathcal{P}=\left\{\mathcal{P}_{n}\right\}_{n} \geqslant 1$ in $\mathcal{C}$ together with linear maps

$$
\circ_{i}: \mathcal{P}_{n} \otimes \mathcal{P}_{m} \rightarrow \mathcal{P}_{n+m-1},
$$

one for each $n, m \geqslant 1$, and $1 \leqslant i \leqslant n$, and a distinguished element $\mathbf{1} \in \mathcal{P}_{1}$ such that the following relations hold for all $x \in \mathcal{P}_{n}, y \in \mathcal{P}_{m}$, and $z \in \mathcal{P}_{p}$ :

$$
\begin{aligned}
\left(x \circ_{j} z\right) \circ_{i} y & =\left(x \circ_{i} y\right) \circ_{m+j-1} z, & & \text { if } 1 \leqslant i<j \leqslant n, \\
\left(x \circ_{i} y\right) \circ_{i+j-1} z & =x \circ_{i}\left(y \circ_{j} z\right), & & \text { if } 1 \leqslant i \leqslant n \text { and } 1 \leqslant j \leqslant m, \\
x \circ_{i} \mathbf{1} & =x, & & \text { if } 1 \leqslant i \leqslant n, \\
\mathbf{1} \circ_{1} x & =x . & &
\end{aligned}
$$

For an equivalent definition of non-symmetric operads, see [10]. This uses maps

$$
\gamma: \mathcal{P}_{k} \otimes \mathcal{P}_{i_{1}} \otimes \cdots \otimes \mathcal{P}_{i_{k}} \rightarrow \mathcal{P}_{i_{1}+\cdots+i_{k}}
$$

which satisfy certain natural associativity axioms. To go from one definition to the other it is enough to know that

$$
\gamma\left(x ; y_{1}, \ldots, y_{k}\right)=\left(\cdots\left(\left(\left(x \circ_{1} y_{1}\right) \circ_{i_{1}+1} y_{2}\right) \circ_{i_{1}+i_{1}+1} y_{3}\right) \cdots\right)
$$

and

$$
x \circ_{i} y=\gamma(x ; 1, \ldots, 1, y, 1, \ldots, 1),
$$

where $y$ is in the $i^{t h}$ position.

For $\mathcal{P}$ as above we denote by $\operatorname{deg} x$ the degree of an element $x \in \mathcal{P}$ and by $|x|$ the degree in desuspension. That is, if $x \in \mathcal{P}_{n}$ then $\operatorname{deg} x=n$ and $|x|=n-1$.

In [7], for each unital operad of vector spaces (symmetric or not), Gerstenhaber and Voronov defined the following collection of multilinear operations (braces on $\mathcal{P}$ ):

$$
x\left\{x_{1}, \ldots, x_{n}\right\}:=\sum(-1)^{\epsilon} \gamma\left(x ; i d, \ldots, i d, x_{1}, i d, \ldots, i d, x_{n}, i d, \ldots, i d\right)
$$

for $x, x_{1}, \ldots, x_{n} \in \mathcal{P}$, where the summation goes through all possible substitutions of $x_{1}, \ldots, x_{n}$ into $x$ in the prescribed order and $\epsilon=\sum_{i=1}^{n}\left|x_{p}\right| i_{p}$, where $i_{p}$ is the total number of inputs in front of $x_{p}$. With the conventions $x\{\}:=x$ and $x \circ y:=x\{y\}$, Gerstenhaber and Voronov proved that the braces satisfy certain identities, which in particular imply that the degree -1 bracket

$$
[x, y]:=x \circ y-(-1)^{|x||y|} y \circ x
$$

defines a structure of a graded Lie algebra on $\mathcal{P}$. 
A multiplication on an operad $\mathcal{P}$ is an element $m \in \mathcal{P}_{2}$ such that $m \circ m=0$. Such a multiplication defines a differential graded associative algebra on $\mathcal{P}$ as follows (see [7], Proposition 2). Set the product

$$
x \cdot y:=(-1)^{|x|+1} m\{x, y\}
$$

and the differential

$$
d x=m \circ x-(-1)^{|x|} x \circ m, \quad d^{2}=0, \quad \operatorname{deg} d=1 .
$$

Gerstenhaber and Voronov proved that a multiplication on an operad of vector spaces implies the existence of even more algebraic structure on the operad. More precisely, they proved the following theorem.

Theorem 2.4. A multiplication on an operad $\mathcal{P}=\left\{\mathcal{P}_{n}\right\}_{n} \geqslant 1$ defines the structure of a homotopy $G$-algebra on $\mathcal{P}=\oplus \mathcal{P}_{n}$.

The precise definition of a homotopy $G$-algebra can be found in [7]. As noted in the same paper, every homotopy $G$-algebra is a differential graded Lie algebra with respect to the commutator $[-,-]$, which is a graded derivation of the dot product up to null-homotopy. This implies that we have the following identities:

$$
\begin{aligned}
{[x, y \cdot z]-[x, y] \cdot z-(-1)^{|x|(|y|+1)} y \cdot[x, z] } \\
=(-1)^{|x|+|y|+1}\left(d(x\{y, z\})-(d x)\{y, z\}-(-1)^{|x|} x\{d y, z\}-(-1)^{|x|+|y|} x\{y, d z\}\right) \\
\quad x \cdot y-(-1)^{(|x|+1)(|y|+1)} y \cdot x=(-1)^{|x|}\left(d(x \circ y)-d x \circ y-(-1)^{|x|} x \circ d y\right) .
\end{aligned}
$$

Note that the multiplication is homotopy graded commutative.

We conclude this section by recalling the definition of a $G$-algebra.

Definition 2.5. A $G$-algebra is a graded vector space $H$ together with a dot product $x \cdot y$ defining the structure of a graded commutative algebra and with a bracket $[x, y]$ of degree -1 defining the structure of a graded Lie algebra, such that the bracket with an element is a derivation of the dot product:

$$
[x, y \cdot z]=[x, y] \cdot z+(-1)^{|x|(|y|+1)} y \cdot[x, z] .
$$

\section{The operad structure on $C^{*}((A, B, \varepsilon) ; A)$}

In this section we define a multiplicative non-symmetric unital operad structure on $C^{*}((A, B, \varepsilon) ; A)$. The results of $[\mathbf{7}]$, outlined in Section 2.3 , imply the existence of a homotopy $G$-algebra structure on $C^{*}((A, B, \varepsilon) ; A)$. In particular, the secondary Hochschild cohomology $H^{*}((A, B, \varepsilon) ; A)$ has a natural $G$-algebra structure.

Let $\mathcal{P}_{n}:=C^{n}((A, B, \varepsilon) ; A)$, for $n \geqslant 1$. For each $m, n \geqslant 1$ and $1 \leqslant i \leqslant n$ we define the linear maps

$$
\circ_{i}: \mathcal{P}_{n} \otimes \mathcal{P}_{m} \rightarrow \mathcal{P}_{n+m-1}
$$


as follows: If $f^{n} \in \mathcal{P}_{n}, g^{m} \in \mathcal{P}_{m}$ and $1 \leqslant i \leqslant n$ then

$$
\begin{aligned}
& f^{n} \circ_{i} g^{m}\left(\otimes\left(\begin{array}{ccccc}
a_{1} & b_{1,2} & \cdots & b_{1, n+m-2} & b_{1, n+m-1} \\
1 & a_{2} & \cdots & b_{2, n+m-2} & b_{2, n+m-1} \\
\vdots & \vdots & & \vdots & \vdots \\
1 & 1 & \cdots & a_{n+m-2} & b_{n+m-2, n+m-1} \\
1 & 1 & \cdots & 1 & a_{n+m-1}
\end{array}\right)\right) \\
& \left.=f^{n}\left(\begin{array}{ccccccc}
a_{1} & \cdots & b_{1, i-1} & \prod_{j=i}^{m+i-1} b_{1, j} & b_{1, m+i} & \cdots & b_{1, n+m-1} \\
& & & & & \\
1 & \cdots & b_{2, i-1} & \prod_{j=i}^{m+i-1} b_{2, j} & b_{2, m+i} & \cdots & b_{2, n+m-1} \\
\vdots & & \vdots & \vdots & \vdots & & \vdots \\
1 & \cdots & a_{i-1} & \prod_{j=i}^{m+i-1} b_{i-1, j} & b_{i-1, m+i} & \cdots & b_{i-1, n+m-1} \\
& & & & & & \\
1 & \cdots & 1 & g^{m}\left(T_{m+i-1}^{i-1}\right) & \prod_{j=i}^{m+i-1} b_{j, m+i} & \cdots & \prod_{j=i}^{m+i-1} b_{j, n+m-1} \\
& \cdots & 1 & 1 & a_{m+i} & \cdots & b_{m+i, n+m-1} \\
\vdots & & \vdots & \vdots & \vdots & & \vdots \\
1 & \cdots & 1 & 1 & 1 & \cdots & b_{n+m-2, n+m-1} \\
1 & \cdots & 1 & 1 & 1 & \cdots & a_{n+m-1}
\end{array}\right)\right)
\end{aligned}
$$

It is a straightforward computation to show that the maps " $\circ_{i}$ " satisfy the equations of Definition 2.3, where the unit of the operad is the identity map $A \rightarrow A$. We will only check here that, for $1 \leqslant i<j \leqslant n$, we have

$$
\left(f^{n} \circ_{j} h^{p}\right) \circ_{i} g^{m}=\left(f^{n} \circ_{i} g^{m}\right) \circ_{m+j-1} h^{p}
$$

and leave the remaining three relations to the reader. Indeed, we have that

$$
\begin{aligned}
& \left(f^{n} \circ_{j} h^{p}\right) \circ_{i} g^{m}\left(\otimes\left(\begin{array}{ccc}
a_{1} & \cdots & b_{1, m+n+p-2} \\
\vdots & & \vdots \\
1 & \cdots & a_{m+n+p-2}
\end{array}\right)\right) \\
& \left.=\left(f^{n} \circ_{j} h^{p}\right)\left(\begin{array}{ccccc}
a_{1} & \cdots & \prod_{k=i}^{i+m-1} b_{1, k} & \cdots & b_{1, m+n+p-2} \\
\vdots & & \vdots & & \vdots \\
1 & \cdots & g^{m}\left(T_{m+i-1}^{i-1}\right) & \cdots & \prod_{k=i}^{i+m-1} b_{k, m+n+p-2} \\
\vdots & & \vdots & & \vdots \\
1 & \cdots & 1 & \cdots & a_{m+n+p-2}
\end{array}\right)\right)
\end{aligned}
$$


OPERATIONS ON THE SECONDARY HOCHSCHILD COHOMOLOGY

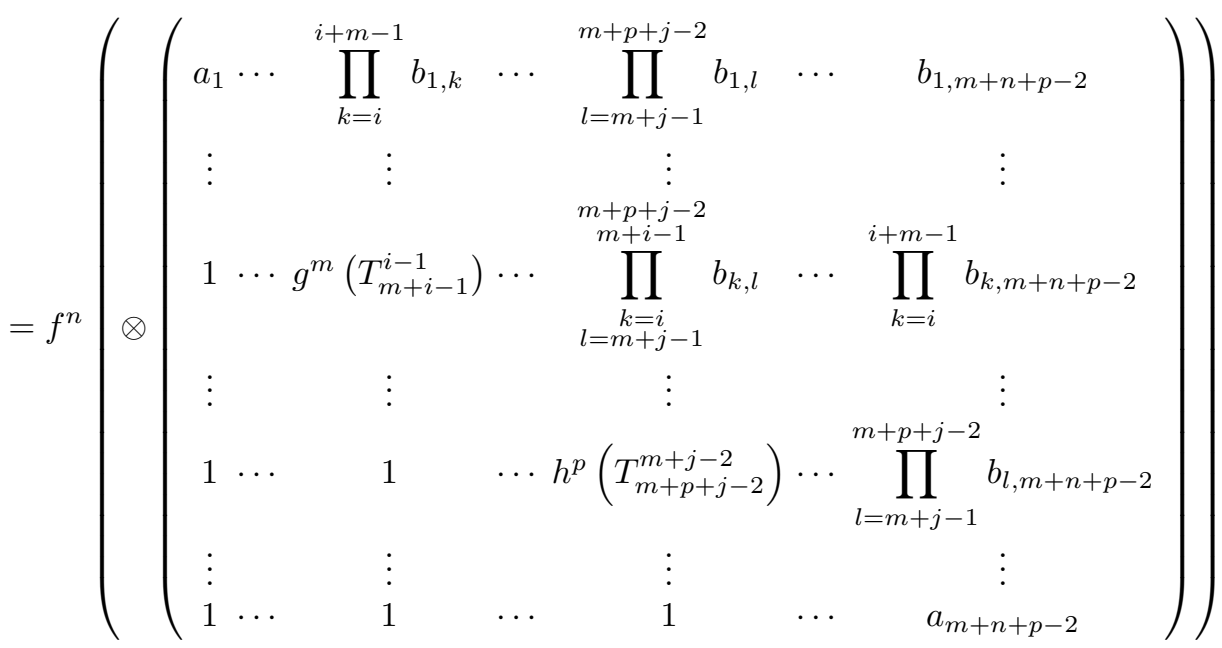

$$
\begin{aligned}
& =\left(f^{n} \circ_{i} g^{m}\right)\left(\otimes\left(\begin{array}{ccccc}
a_{1} & \cdots & \prod_{l=m+j-1}^{m+p+j-2} b_{1, l} & \cdots & b_{1, m+n+p-2} \\
\vdots & & \vdots & & \vdots \\
1 & \cdots & h\left(T_{m+p+j-2}^{m+j-2}\right) & \cdots & \prod_{l=m+j-1}^{m+p+j-2} b_{l, m+n+p-2} \\
\vdots & & \vdots & & \vdots \\
1 & \cdots & 1 & \cdots & a_{m+n+p-2}
\end{array}\right)\right) \\
& =\left(f^{n} \circ_{i} g^{m}\right) \circ_{m+j-1} h^{p}\left(\otimes\left(\begin{array}{ccc}
a_{1} & \cdots & b_{1, m+n+p-2} \\
\vdots & & \vdots \\
1 & \cdots & a_{m+n+p-2}
\end{array}\right)\right) \text {. }
\end{aligned}
$$

As noted in Section 2.3, an operad structure on $C^{n}((A, B, \varepsilon) ; A)$ implies the existence of a graded Lie algebra structure on $\oplus_{n} \geqslant 1 C^{n}((A, B, \varepsilon) ; A)$. More explicitly, Equation 1 defines a degree -1 bracket as follows:

$$
\left[f^{n}, g^{m}\right]=f^{n} \circ g^{m}-(-1)^{(n-1)(m-1)} g^{m} \circ f^{n},
$$

where $f^{n} \circ g^{m}=f^{n}\left\{g^{m}\right\}=\sum_{i=1}^{n}(-1)^{(i-1)(m-1)} f^{n} \circ_{i} g^{m}$.

In addition, one can see that the non-symmetric operad $\left\{\mathcal{P}_{n}\right\}_{n \geqslant 1}$ is multiplicative. Indeed, the map $\pi \in \mathcal{P}_{2}=C^{2}((A, B, \varepsilon) ; A)$ defined by

$$
\pi\left(\otimes\left(\begin{array}{cc}
a & \alpha \\
1 & b
\end{array}\right)\right)=\varepsilon(\alpha) a b
$$

satisfies $\pi \circ \pi=0$.

The existence of a multiplication on $\left\{\mathcal{P}_{n}\right\}_{n} \geqslant 1$ determines a much richer algebraic structure on the operad. Using Proposition 2 and Theorem 3 of [7] (outlined in Section 2.3) we obtain the following result. 
Proposition 3.1. If $A, B$ are associative algebras over a field $k, B$ is commutative, and $\varepsilon: B \rightarrow A$ is a morphism of algebras whose image belongs to the center of $A$, then the secondary Hochschild complex $C^{*}((A, B, \varepsilon) ; A)$ has a natural structure of homotopy G-algebra.

One can notice that for $f^{n} \in C^{n}((A, B, \varepsilon) ; A)$ and $g^{m} \in C^{m}((A, B, \varepsilon) ; A)$ the $\operatorname{dot}$ product is given by

$$
\begin{aligned}
& f^{n} \cdot g^{m}\left(\otimes\left(\begin{array}{ccccc}
a_{1} & b_{1,2} & \cdots & b_{1, n+m-1} & b_{1, n+m} \\
1 & a_{2} & \cdots & b_{2, n+m-1} & b_{2, n+m} \\
\vdots & \vdots & & \vdots & \vdots \\
1 & 1 & \cdots & a_{n+m-1} & b_{n+m-1, n+m} \\
1 & 1 & \cdots & 1 & a_{n+m}
\end{array}\right)\right) \\
& =(-1)^{n m} f^{n}\left(\otimes\left(\begin{array}{ccccc}
a_{1} & b_{1,2} & \cdots & b_{1, n-1} & b_{1, n} \\
1 & a_{2} & \cdots & b_{2, n-1} & b_{2, n} \\
\vdots & \vdots & & \vdots & \vdots \\
1 & 1 & \cdots & a_{n-1} & b_{n-1, n} \\
1 & 1 & \cdots & 1 & a_{n}
\end{array}\right)\right) \cdot \prod_{\substack{n+1 \leqslant n+m \\
1 \leqslant i \leqslant n}} \varepsilon\left(b_{i, j}\right) \\
& \cdot g^{m}\left(\otimes\left(\begin{array}{ccccc}
a_{n+1} & b_{n+1, n+2} & \cdots & b_{n+1, n+m-1} & b_{n+1, n+m} \\
1 & a_{n+2} & \cdots & b_{n+2, n+m-1} & b_{n+2, n+m} \\
\vdots & \vdots & & \vdots & \vdots \\
1 & 1 & \cdots & a_{n+m-1} & b_{n+m-1, n+m} \\
1 & 1 & \cdots & 1 & a_{n+m}
\end{array}\right)\right) \text {. }
\end{aligned}
$$

A condensed version of the above formula is

$$
\left(f^{n} \cdot g^{m}\right)\left(T_{n+m}^{0}\right)=(-1)^{n m} f\left(T_{n}^{0}\right) g\left(T_{n+m}^{n}\right) \prod_{\substack{n+1 \leqslant j \leqslant n+m \\ 1 \leqslant i \leqslant n}} \varepsilon\left(b_{i, j}\right) .
$$

If $B=k$, the secondary Hochschild cohomology $H^{*}((A, B, \varepsilon) ; A)$ coincides with the Hochschild cohomology $H^{*}(A, A)$. We should note that the product $f^{n} \cdot g^{m}$ is the usual cup product $f^{n} \smile g^{m}$ altered by the sign $(-1)^{n m}$. One can define an extension of this cup product to one on the secondary Hochschild complex by dropping the sign $(-1)^{n m}$ and expect to have the usual properties.

Similarly, we have

$$
d\left(f^{n}\right)=(-1)^{n+1} \delta_{n}^{\varepsilon}\left(f^{n}\right),
$$

where $\delta_{n}^{\varepsilon}$ is the differential of the secondary Hochschild complex, which in the case $B=k$ coincides with the differential $\delta_{n}$ of the Hochschild complex $C^{*}(A, A)$. The sign difference does not change the cohomology, so, by taking into account Proposition 3.1, we obtain the following corollary.

Corollary 3.2. $H^{*}((A, B, \varepsilon) ; A)$ has a natural $G$-algebra structure.

Proof. The proof follows from the above discussion and the results from [7]. We already know that the bracket defines a graded Lie algebra structure on the chain groups $C^{*}((A, B, \varepsilon) ; A)$, one which obviously induces a graded Lie algebra structure on $H^{*}((A, B, \varepsilon) ; A)$. 
In addition, the dot product is well defined on $H^{*}((A, B, \varepsilon) ; A)$. Indeed, for all cochains $y, z \in C^{*}((A, B, \varepsilon) ; A)$ we have that

$$
d(y \cdot z)=(d y) \cdot z+(-1)^{|y|+1} y \cdot(d z) .
$$

The above formula can be verified directly or obtained from Equation 4. Finally, Equation 4 also implies that the bracket is a derivation of the dot product and Equation 5 implies that the dot product is graded commutative.

\section{Extensions of $B$-algebras}

In this section we use the secondary Hochschild cohomology in order to describe a certain class of $B$-algebra extensions.

Suppose that $X$ is a $B$-algebra with $\varepsilon_{X}: B \rightarrow X$ and that there exists a surjective morphism of $B$-algebras $\pi: X \rightarrow A$ such that $\operatorname{ker}(\pi)^{2}=0$. Let $M=\operatorname{ker}(\pi)$. We require that the $B$-algebra structure induced on $A$ by the map $\pi \circ \varepsilon_{X}$ coincides with that defined by the map $\varepsilon$. Consider $s: A \rightarrow X$ a $k$-linear map such that $\pi s=i d_{A}$. Then $M$ is an $A$-bimodule with the multiplication given by

$$
a m=s(a) m, \quad m a=m s(a),
$$

for all $m \in M$ and $a \in A$. One can notice that this action does not depend on the choice of the section $s$. Moreover, for all $\alpha \in B$ and all $m \in M$ we have

$$
\varepsilon(\alpha) m=m \varepsilon(\alpha)=\varepsilon_{X}(\alpha) m .
$$

As a $k$-vector space we obviously have that $X=s(A) \oplus M$ (that is, $s(A)+M=X$ and $s(A) \cap M=0)$.

Because of Proposition 2.1 from [12], we know that a $B$-algebra structure on $X$ is the same as an associative family of products $m_{\alpha, X}: X \otimes X \rightarrow X$ where $m_{\alpha, X}(x \otimes$ $y)=\varepsilon_{X}(\alpha) x y$. Since $\pi: X \rightarrow A$ is a morphism of $B$-algebras we must have that

$$
\pi\left(m_{\alpha, X}((s(a)+m) \otimes(s(b)+n))\right)=m_{\alpha}(\pi(s(a)+m) \otimes \pi(s(b)+n))=\varepsilon(\alpha) a b .
$$

Using this and the linearity of the product we get

$$
\begin{aligned}
& m_{\alpha, X}((s(a)+m) \otimes(s(b)+n))= \\
& \varepsilon_{X}(\alpha)(s(a) s(b)+s(a) n+m s(b))= \\
& s(\varepsilon(\alpha) a b)+\varepsilon(\alpha) a n+m b \varepsilon(\alpha)+\varepsilon_{X}(\alpha) s(a) s(b)-s(\varepsilon(\alpha) a b) .
\end{aligned}
$$

One can see that the $k$-linear map $c_{s}: A \otimes A \otimes B \rightarrow M$ defined by

$$
c_{s}(a \otimes b \otimes \alpha)=\varepsilon_{X}(\alpha) s(a) s(b)-s(\varepsilon(\alpha) a b)
$$

is a 2-cocycle. Moreover, if $t: A \rightarrow X$ is another section for $\pi$, then

$$
\delta_{1}^{\varepsilon}(s-t)=c_{s}-c_{t} .
$$

To summarize, we have the following result:

Lemma 4.1. Let $X$ be a $B$-algebra and $\pi: X \rightarrow A$ a surjective morphism of $B$ algebras such that $M^{2}=0$ (where $M=k e r(\pi)$ ). Then $\widehat{c_{s}} \in H^{2}((A, B, \varepsilon) ; M)$ does not depend on the choice of the section $s$. We will denote this element by $c_{X, \pi}$. 
Next we prove that $c_{X, \pi}$ depends only on the isomorphism class of the extension $0 \rightarrow M \rightarrow X \stackrel{\pi}{\rightarrow} A \rightarrow 0$.

Proposition 4.2. Let $X_{1}$ and $X_{2}$ be two B-algebras, $\pi_{i}: X_{i} \rightarrow A$ surjective morphisms of B-algebras such that $\left(k e r\left(\pi_{i}\right)\right)^{2}=0$. Moreover, assume that there exists an isomorphism of $B$-algebras $F: X_{1} \rightarrow X_{2}$ such that $\pi_{2} \circ F=\pi_{1}$. Under the identification $M_{2}=\operatorname{ker}\left(\pi_{2}\right)=F\left(M_{1}\right)$ we have that $c_{X_{2}, \pi_{2}}=F^{*}\left(c_{X_{1}, \pi_{1}}\right) \in H^{2}\left((A, B, \varepsilon) ; M_{2}\right)$.

Proof. The proof follows from Lemma 4.1 and the fact that if $s: A \rightarrow X_{1}$ is a section for $\pi_{1}$ then Fs: $A \rightarrow X_{2}$ is a section for $\pi_{2}$.

In addition, for any $A$-bimodule $M$ such that $\varepsilon(\alpha) m=m \varepsilon(\alpha)$ and for any cocycle $c \in C^{2}((A, B, \varepsilon) ; M)$ we can define a $B$-algebra $X$ and a surjective morphism of $B$ algebras $\pi: X \rightarrow A$ such that $M=\operatorname{ker}(\pi), M^{2}=0$, and $\pi \circ \varepsilon_{X}=\varepsilon$. To see this we use Proposition 2.1 from [12] to define a family of products $m_{\alpha, X}: X \otimes X \rightarrow X$ as follows. First, we take $X=A \oplus M$, as a $k$-vector space. Second, we define

$$
m_{\alpha, X}((a+m) \otimes(b+n))=\varepsilon(\alpha) a b+\varepsilon(\alpha) a n+m b \varepsilon(\alpha)+c(a \otimes b \otimes \alpha) .
$$

One can check without any difficulty that $\left(X, m_{1, X}\right)$ is a $k$-algebra, with unit $1_{X}=$ $1_{A}-c\left(1_{A} \otimes 1_{A} \otimes 1_{B}\right)$, and that for all $\alpha, \beta \in B$ and $q \in k$ we have $m_{\alpha+\beta, X}=m_{\alpha, X}+$ $m_{\beta, X}$ and $m_{q \alpha, X}=q m_{\alpha, X}$. The third condition of Proposition 2.1, $m_{\beta \gamma, X}\left(m_{\alpha, X} \otimes\right.$ $i d)=m_{\alpha \beta, X}\left(i d \otimes m_{\gamma, X}\right)$, is equivalent to $c$ being a cocycle and it is satisfied, so $X$ is a $B$-algebra. We have $\varepsilon_{X}: B \rightarrow X$ defined by

$$
\varepsilon_{X}(\alpha)=\varepsilon(\alpha)-2 \varepsilon(\alpha) c\left(1_{A} \otimes 1_{A} \otimes 1_{B}\right)+c\left(1_{A} \otimes 1_{A} \otimes \alpha\right) .
$$

Third, it is clear that the canonical projection $\pi: X \rightarrow A$ is a surjective morphism of $k$-algebras such that $\operatorname{ker}(\pi)=M, M^{2}=0$, and that $\pi \circ \varepsilon_{X}(\alpha)=\varepsilon(\alpha)$. To see that $\pi$ is a morphism of $B$-algebras note that for all $\alpha \in B, a \in A$, and $m \in M$ we have

$$
\begin{aligned}
\pi(\alpha(a+m)) & =\pi\left(m_{1, X}\left(\varepsilon_{X}(\alpha) \otimes(a+m)\right)\right) \\
& =\pi\left(m_{1, X}\left(\left(\varepsilon(\alpha)-2 \varepsilon(\alpha) c\left(1_{A} \otimes 1_{A} \otimes 1_{B}\right)+c\left(1_{A} \otimes 1_{A} \otimes \alpha\right)\right) \otimes(a+m)\right)\right) \\
& =\varepsilon(\alpha) a \\
& =\alpha \pi(a+m) .
\end{aligned}
$$

Finally, we show that the construction of $X$ depends only on the cohomology class of the cocycle $c \in C^{2}((A, B, \varepsilon) ; M)$. For this let $c_{1}, c_{2} \in C^{2}((A, B, \varepsilon) ; M)$ such that $c_{1}-c_{2}=\delta_{1}^{\varepsilon} f$, where $f: A \rightarrow M$ is $k$-linear. Denote by $X_{1}$ and $X_{2}$ the $B$-algebras defined by the cocycles $c_{1}$ and $c_{2}$, by $m_{\alpha, X_{1}}$ and $m_{\alpha, X_{2}}$ their corresponding families of products, and by $\pi_{1}$ and $\pi_{2}$ the canonical projections of $X_{1}$ and $X_{2}$ onto $A$. Note that by construction $X_{1}=X_{2}=A \oplus M$ as $k$-vector spaces. Then the map $F: X_{1} \rightarrow X_{2}$, defined by the formula $F(a+m)=a+m+f(a)$, is an isomorphism of $B$-algebras such that $\pi_{2} \circ F=\pi_{1}$. It is easy to see that $F$ is an isomorphism of $k$-algebras such that $\pi_{2} \circ F=\pi_{1}$, so we will only prove that $F$ is $B$-linear. Indeed, for $\alpha \in B, a \in A$, and $m \in M$ we have

$$
\begin{aligned}
F(\alpha(a+m))= & F\left(m_{1, X_{1}}\left(\varepsilon_{X_{1}}(\alpha) \otimes(a+m)\right)\right) \\
= & F\left(m_{1, X_{1}}\left(\left(\varepsilon(\alpha)-2 \varepsilon(\alpha) c_{1}(1 \otimes 1 \otimes 1)+c_{1}(1 \otimes 1 \otimes \alpha)\right) \otimes(a+m)\right)\right) \\
= & F\left(\varepsilon(\alpha) a+\varepsilon(\alpha) m-2 \varepsilon(\alpha) c_{1}(1 \otimes 1 \otimes 1) a+c_{1}(1 \otimes 1 \otimes \alpha) a\right. \\
& \left.+c_{1}(\varepsilon(\alpha) \otimes a \otimes 1)\right) \\
= & \varepsilon(\alpha) a+\varepsilon(\alpha) m-2 \varepsilon(\alpha) c_{1}(1 \otimes 1 \otimes 1) a+c_{1}(1 \otimes 1 \otimes \alpha) a \\
& +c_{1}(\varepsilon(\alpha) \otimes a \otimes 1)+f(\varepsilon(\alpha) a)
\end{aligned}
$$


and

$$
\begin{aligned}
\alpha F(a+m)= & m_{1, X_{2}}\left(\varepsilon_{X_{2}}(\alpha) \otimes(a+m+f(a))\right) \\
= & m_{1, X_{2}}\left(\left(\varepsilon(\alpha)-2 \varepsilon(\alpha) c_{2}(1 \otimes 1 \otimes 1)+c_{2}(1 \otimes 1 \otimes \alpha)\right) \otimes(a+m+f(a))\right) \\
= & \varepsilon(\alpha) a+\varepsilon(\alpha) m+\varepsilon(\alpha) f(a)-2 \varepsilon(\alpha) c_{2}(1 \otimes 1 \otimes 1) a+c_{2}(1 \otimes 1 \otimes \alpha) a \\
& +c_{2}(\varepsilon(\alpha) \otimes a \otimes 1) .
\end{aligned}
$$

Thus we get

$$
\begin{aligned}
F(\alpha(a+m))-\alpha F(a+m)= & 2 \varepsilon(\alpha)\left[c_{2}(1 \otimes 1 \otimes 1)-c_{1}(1 \otimes 1 \otimes 1)\right] a \\
& +\left[c_{1}(1 \otimes 1 \otimes \alpha)-c_{2}(1 \otimes 1 \otimes \alpha)\right] a \\
& +\left[c_{1}(\varepsilon(\alpha) \otimes a \otimes 1)-c_{2}(\varepsilon(\alpha) \otimes a \otimes 1)\right] \\
& -\varepsilon(\alpha) f(a)+f(\varepsilon(\alpha) a) .
\end{aligned}
$$

Since $c_{1}-c_{2}=\delta_{1}^{\varepsilon} f$ we have the following identities:

$$
\begin{aligned}
c_{2}(1 \otimes 1 \otimes 1)-c_{1}(1 \otimes 1 \otimes 1) & =-f(1) \\
c_{1}(1 \otimes 1 \otimes \alpha)-c_{2}(1 \otimes 1 \otimes \alpha) & =2 \varepsilon(\alpha) f(1)-f(\varepsilon(\alpha)) \\
c_{1}(\varepsilon(\alpha) \otimes a \otimes 1)-c_{2}(\varepsilon(\alpha) \otimes a \otimes 1) & =\varepsilon(\alpha) f(a)-f(\varepsilon(\alpha) a)+f(\varepsilon(\alpha)) a .
\end{aligned}
$$

Therefore we obtain that $F(\alpha(a+m))-\alpha F(a+m)=0$, so $F$ is an isomorphism of $B$-algebras such that $\pi_{2} \circ F=\pi_{1}$.

Assume now that we have an extension given by the following data: a morphism of $k$-algebras $\varepsilon_{X}: B \rightarrow X$; a surjective morphism of $B$-algebras $\pi: X \rightarrow A$ such that $\operatorname{ker}(\pi)^{2}=0, M=\operatorname{ker}(\pi) ; \pi \circ \varepsilon_{X}=\varepsilon$; and a $k$-linear map $s: A \rightarrow X$ such that $\pi s=i d_{A}$. If we consider the cocycle $c_{s} \in C^{2}((A, B, \varepsilon) ; M)$ defined earlier and then we consider the extension associated to this cocycle then it is not hard to see that we obtain an extension equivalent to the initial one. Similarly, given an $A$-bimodule $M$ such that $\varepsilon(a) m=m \varepsilon(a)$ and a cocycle $c \in C^{2}((A, B, \varepsilon) ; M)$ we construct the extension associated to $c$. If we now take the cocycle $c_{s}$ determined by a section $s: A \rightarrow X$ with $\pi s=i d_{A}$ then we have that $c_{s}-c=\delta_{1}^{\varepsilon} u$, where $u: A \rightarrow M$ is the $k$-linear map induced by $s$ on $M$. Indeed, we have that $c_{s}(a \otimes b \otimes \alpha)=c(\varepsilon(\alpha) \otimes a b \otimes 1)+$ $c(1 \otimes 1 \otimes \alpha) a b-2 \varepsilon(\alpha) c(1 \otimes 1 \otimes 1) a b+\varepsilon(\alpha) c(a \otimes b \otimes 1)+\delta u(a \otimes b \otimes \alpha)$ for all $a, b \in$ $A$ and $\alpha \in B$. The key observation here is that the cocycle condition implies that $c(a \otimes$ $b \otimes \alpha)=c(\varepsilon(\alpha) \otimes a b \otimes 1)+c(1 \otimes 1 \otimes \alpha) a b-2 \varepsilon(\alpha) c(1 \otimes 1 \otimes 1) a b+\varepsilon(\alpha) c(a \otimes b \otimes 1)$.

The above considerations allow us to conclude that $H^{2}((A, B, \varepsilon) ; M)$ can be naturally identified with the equivalence classes of extensions of $B$-algebras of $A$ by $M$, for any $A$-bimodule $M$ such that $\varepsilon(\alpha) m=m \varepsilon(\alpha)$.

\section{A Hodge-type decomposition of the secondary cohomology}

In this section we will assume that $A$ is commutative, $k$ is a field of characteristic 0 , and $M$ is a symmetric $A$-bimodule (i.e. $a m=m a$ for all $a \in A$ and $m \in M$ ). We denote by $k S_{n}$ the group algebra of the group of permutations of $n$ objects. Under these conditions Barr proved in $[\mathbf{2}]$ that $k S_{n}$ operates on the $n$-cochains, $C^{n}(A, M)$, of the complex defining the Hochschild cohomology of $A$ with coefficients in $M$ and that there is a non-central idempotent $e_{n} \in \mathbb{Q} S_{n}$ such that $\delta_{n}\left(e_{n} f\right)=e_{n+1}\left(\delta_{n} f\right)$. This implies that the Hochschild complex is a direct sum of two sub-complexes, corresponding to $e_{n}$ and $1-e_{n}$. Barr's ideas were extended in $[\mathbf{6}]$ by Gerstenhaber and Schack, who showed that $\mathbb{Q} S_{n}$ contains $n$ mutually orthogonal idempotents 
$e_{n}(1), e_{n}(2), \ldots, e_{n}(n)$ which sum to the identity and with the property that for each cochain $f \in C^{n}(A, M)$ we have $\delta_{n}\left(e_{n}(k) f\right)=e_{n+1}(k)\left(\delta_{n} f\right)$. From this it follows that the Hochschild cohomology $H^{n}(A, M)$ has a Hodge-type decomposition into a direct sum of $n$ summands. Barr's original idempotent $e_{n}$ is $e_{n}(1)$ and the idempotents and the decomposition are labeled BGS (Barr-Gerstenhaber-Schack). The action of $S_{n}$ on the $n$-cochains $C^{n}(A, M)$ is given by

$$
(\pi f)\left(a_{1} \otimes \cdots \otimes a_{n}\right)=\left(f \pi^{-1}\right)\left(a_{1} \otimes \cdots \otimes a_{n}\right)=f\left(a_{\pi(1)} \otimes a_{\pi(2)} \otimes \cdots \otimes a_{\pi(n)}\right) .
$$

It is not hard to see that $S_{n}$ acts on the $n$-cochains of the secondary cohomology. Indeed, for $\pi \in S_{n}$ and $f \in C^{n}((A, B, \varepsilon) ; M)$ we define the left action of $S_{n}$ by setting

$$
(\pi f)\left(\otimes\left(\begin{array}{cccc}
a_{1} & b_{1,2} & \cdots & b_{1, n} \\
1 & a_{2} & \cdots & b_{2, n} \\
\vdots & \vdots & & \vdots \\
1 & 1 & \cdots & a_{n}
\end{array}\right)\right)=f\left(\otimes\left(\begin{array}{cccc}
a_{\pi(1)} & b_{\pi(1,2)} & \cdots & b_{\pi(1, n)} \\
1 & a_{\pi(2)} & \cdots & b_{\pi(2, n)} \\
\vdots & \vdots & & \vdots \\
1 & 1 & \cdots & a_{\pi(n)}
\end{array}\right)\right)
$$

where, for each $1 \leqslant i<j \leqslant n$, the element $b_{\pi(i, j)}$ is equal to $b_{\pi(i), \pi(j)}$ if $\pi(i)<\pi(j)$ and equal to $b_{\pi(j), \pi(i)}$ if $\pi(j)<\pi(i)$. Similarly, one defines the right action of $S_{n}$ on $C^{n}((A, B, \varepsilon) ; M)$ by using $\pi^{-1}$. It is important to note that the order of the elements $a_{\pi(1)}, a_{\pi(2)}, \ldots, a_{\pi(n)}$ on the diagonal of the above tensor matrix determines completely the positions of $b_{\pi(i, j)}$.

We want to show that for $f \in C^{n}((A, B, \varepsilon) ; M)$ we have that

$$
\delta_{n}^{\varepsilon}\left(e_{n}(k) f\right)=e_{n+1}(k)\left(\delta_{n}^{\varepsilon} f\right) .
$$

This will imply that the secondary cohomology $H^{\bullet}((A, B, \varepsilon) ; M)$ has a Hodge-type decomposition. For this we use the fact that the BGS idempotents $e_{n}(1), e_{n}(1), \ldots, e_{n}(n)$ are polynomials, with rational coefficients, of the total shuffle operator.

Following Barr [2], for $0<r<n$ and $\pi \in S_{n}$ we say that $\pi$ is a pure shuffle of $r$ through $n-r$ if $\pi(1)<\cdots<\pi(r)$ and $\pi(r+1)<\cdots<\pi(n)$. Then the $r^{\text {th }}$ shuffle operator is $s_{r, n-r}=\sum_{\text {pure }}(-1)^{\pi} \pi$, where $(-1)^{\pi}$ is the sign of $\pi$. The total shuffle operator is defined by $s_{n}=\sum_{1 \leqslant r \leqslant n-1} s_{r, n-r}$ and satisfies $\delta_{n}\left(s_{n} f\right)=s_{n+1}\left(\delta_{n} f\right)$, for all $f \in C^{n}(A, M)$. Moreover, Gerstenhaber and Schack showed in [6] that the minimal polynomial of $s_{n}$ over $\mathbb{Q}$ is $\mu_{n}(x)=\prod_{1 \leqslant i \leqslant n}\left[x-\left(2^{i}-2\right)\right]$. They defined

$$
e_{n}(k)=\prod_{\substack{1 \leqslant i \leqslant n \\ i \neq k}}\left(\lambda_{k}-\lambda_{i}\right)^{-1} \prod_{\substack{1 \leqslant i \leqslant n \\ i \neq k}}\left(s_{n}-\lambda_{i}\right), \text { where } \lambda_{i}=2^{i}-2 .
$$

We want to prove that for every $f \in C^{n}((A, B, \varepsilon), M)$ we have

$$
\left(\delta_{n}^{\varepsilon}\left(s_{n} f\right)-s_{n+1}\left(\delta_{n}^{\varepsilon} f\right)\right)\left(\left(\begin{array}{cccc}
a_{1} & b_{1,2} & \cdots & b_{1, n+1} \\
1 & a_{2} & \cdots & b_{2, n+1} \\
\vdots & \vdots & & \vdots \\
1 & 1 & \cdots & a_{n+1}
\end{array}\right)\right)=0
$$

for $a_{1}, a_{2}, \ldots, a_{n+1} \in A$ and $b_{i j} \in B, 1 \leqslant i<j \leqslant n+1$. The expansion of the left 
side shows that the identity holds for $b_{i, j}=1$, a direct consequence of $\delta_{n}\left(s_{n} \bar{f}\right)-$ $s_{n+1}\left(\delta_{n} \bar{f}\right)=0$ (where $\bar{f}$ is obtained from $f$ by taking $b_{i, j}=1$ ). This means that the diagonals of the tensor sub-matrices of types $T_{1}^{n+1}, T_{n}^{0}$, and $M_{i, i+1}^{1, n}$ in the expansion of $\delta_{n}^{\varepsilon}\left(s_{n} f\right)-s_{n+1}\left(\delta_{n}^{\varepsilon} f\right)$ appear in identical pairs and with opposite signs. But, as a consequence of the way we defined the action of $S_{n}$ on the secondary cochains and of the definition of $\delta_{n}^{\varepsilon}$, the order of the elements $a_{\pi(1)}, a_{\pi(2)}, \ldots, a_{\pi(n+1)}$ and of the products $a_{\pi(i)} a_{\pi(j)} \varepsilon\left(b_{\pi(i, j)}\right)$ on the diagonal of the above tensor matrices determines completely the positions of all $b_{\pi(i, j)}$ and their products in $T_{1}^{n+1}, T_{n}^{0}$, and $M_{i, i+1}^{1, n}$. This implies that $\delta_{n}^{\varepsilon}\left(s_{n} f\right)=s_{n+1}\left(\delta_{n}^{\varepsilon} f\right)$.

In addition, because $\mu_{n}\left(s_{n}\right)=0$, we have the identity

$$
\delta_{n}^{\varepsilon}\left(\mu_{n}\left(s_{n}\right) f\right)=\prod_{1 \leqslant i \leqslant n}\left(s_{n+1}-\lambda_{i}\right)\left(\delta_{n}^{\varepsilon} f\right)=0,
$$

so we get that

$$
\begin{aligned}
\delta_{n}^{\varepsilon}\left(e_{n}(k) f\right) & =\prod_{\substack{1 \leqslant i \leqslant n \\
i \neq k}}\left(\lambda_{k}-\lambda_{i}\right)^{-1} \prod_{\substack{1 \leqslant i \leqslant n \\
i \neq k}}\left(s_{n+1}-\lambda_{i}\right)\left(\delta_{n}^{\varepsilon} f\right) \\
& =\prod_{\substack{1 \leqslant i \leqslant n+1 \\
i \neq k}}\left(\lambda_{k}-\lambda_{i}\right)^{-1} \prod_{\substack{1 \leq i \leqslant n \\
i \neq k}}\left(s_{n+1}-\lambda_{i}\right)\left(\lambda_{k}-\lambda_{n+1}+s_{n+1}-\lambda_{k}\right)\left(\delta_{n}^{\varepsilon} f\right) \\
& =e_{n+1}(k)\left(\delta_{n}^{\varepsilon} f\right) .
\end{aligned}
$$

Adopting the notation from [6], each idempotent $e_{n}(k)$ determines a submodule of $C^{n}((A, B, \varepsilon) ; M)$, namely

$$
C^{k, n-k}((A, B, \varepsilon) ; M)=e_{n}(k) C^{n}((A, B, \varepsilon) ; M) .
$$

By setting $e_{n}(k)=0$ if $k>n, e_{n}(0)=0$ if $n \neq 0$, and $e_{0}(0)=1$ we have that the complex defining the secondary cohomology decomposes as

$$
C^{\bullet}((A, B, \varepsilon) ; M)=\coprod_{k \geqslant 0} C^{k, \bullet-k}((A, B, \varepsilon) ; M)=\coprod_{k \geqslant 0} e_{n}(k) C^{\bullet}((A, B, \varepsilon) ; M) .
$$

Denoting by $H^{k, \bullet-k}((A, B, \varepsilon) ; M)$ the homology of the complex $C^{k, \bullet-k}((A, B, \varepsilon) ; M)$ we have the following

Theorem 5.1. If $\varepsilon: B \rightarrow A$ is a morphism of commutative $k$-algebras, $\mathbb{Q} \subset k$, and $M$ is a symmetric A-bimodule, then

$$
H^{\bullet}((A, B, \varepsilon) ; M)=\coprod_{k \geqslant 0} H^{k, \bullet-k}((A, B, \varepsilon) ; M) .
$$

\section{Some examples}

It was noticed in $[\mathbf{1 2}]$ that there exists a natural morphism

$$
\Phi_{n}: H^{n}((A, B, \varepsilon) ; M) \rightarrow H^{n}(A, M),
$$


induced by the inclusion $i: A^{\otimes n} \rightarrow A^{\otimes n} \otimes B^{\otimes \frac{n(n-1)}{2}}$,

$$
i_{n}\left(a_{1} \otimes \cdots \otimes a_{n}\right)=\otimes\left(\begin{array}{ccccc}
a_{1} & 1 & \cdots & 1 & 1 \\
1 & a_{2} & \cdots & 1 & 1 \\
\vdots & \vdots & & \vdots & \vdots \\
1 & 1 & \cdots & a_{n-1} & 1 \\
1 & 1 & \cdots & 1 & a_{n}
\end{array}\right) .
$$

In this section we will see that in general $\Phi_{n}$ is neither onto nor one to one.

First, notice that if $u: A \rightarrow M$ is $k$-linear such that $\delta_{1}^{\varepsilon}(u)=0$ then we must have that $a \varepsilon(\alpha) u(b)-u(a b \varepsilon(\alpha))+u(a) b \varepsilon(\alpha)=0$. This implies that $\Phi_{1}(u)$ is a derivation that is $B$-linear. Since, in general, not all $k$-derivations of $A$ are $B$-linear we get that $\Phi_{1}$ is not necessarily onto. We have the following result:

\section{Proposition 6.1.}

$$
\begin{gathered}
H^{0}((A, B, \varepsilon) ; M)=M^{A}, \\
H^{1}((A, B, \varepsilon) ; M)=\operatorname{Der}_{B}(A, M) / \operatorname{Inn}(A, M),
\end{gathered}
$$

where $\operatorname{Inn}(A, M)$ denotes the inner derivations.

Proof. Straightforward computation.

Proposition 6.2. Let $\Phi_{2}: H^{2}((A, B, \varepsilon) ; M) \rightarrow H^{2}(A, M)$. If on $M$ we consider the $B$-bimodule structure induced by $\varepsilon$, then there exists an isomorphism

$$
\chi: \frac{\operatorname{Der}_{k}(B, M)}{\varepsilon^{*}\left(\operatorname{Der}_{k}(A, M)\right)} \rightarrow \operatorname{ker}\left(\Phi_{2}\right)
$$

determined by $\chi(u)(a \otimes b \otimes \alpha)=a u(\alpha) b$, for all $a, b \in A$ and $\alpha \in B$.

Proof. Let $\sigma \in Z^{2}((A, B, \varepsilon) ; M)$ such that $\Phi_{2}(\widehat{\sigma})=0 \in H^{2}(A, M)$. This means that there exists a $k$-linear map $u: A \rightarrow M$ such that

$$
\sigma(a \otimes b \otimes 1)=\delta_{1}(u)(a \otimes b)=a u(b)-u(a b)+a u(b) .
$$

We consider the element $\tau \in Z^{2}((A, B, \varepsilon) ; M), \tau=\sigma-\delta_{1}^{\varepsilon}(u)$. Obviously we have that $\widehat{\sigma}=\widehat{\tau} \in H^{2}((A, B, \varepsilon) ; M)$, and $\tau\left(\otimes\left(\begin{array}{cc}a & 1 \\ 1 & b\end{array}\right)\right)=0$.

Since $\tau \in Z^{2}((A, B, \varepsilon) ; M)$, we have

$$
\begin{gathered}
a \varepsilon(\alpha \beta) \tau\left(\otimes\left(\begin{array}{ll}
b & \gamma \\
1 & c
\end{array}\right)\right)-\tau\left(\otimes\left(\begin{array}{cc}
a b \varepsilon(\alpha) & \beta \gamma \\
1 & c
\end{array}\right)\right)+\tau\left(\otimes\left(\begin{array}{cc}
a & \alpha \beta \\
1 & b c \varepsilon(\gamma)
\end{array}\right)\right) \\
-\tau\left(\otimes\left(\begin{array}{cc}
a & \alpha \\
1 & b
\end{array}\right)\right) c \varepsilon(\beta \gamma)=0 .
\end{gathered}
$$

When $\alpha=\beta=1$ we have:

$$
a \tau\left(\otimes\left(\begin{array}{ll}
b & \gamma \\
1 & c
\end{array}\right)\right)=\tau\left(\otimes\left(\begin{array}{cc}
a b & \gamma \\
1 & c
\end{array}\right)\right)
$$

and similarly when $\beta=\gamma=1$,

$$
\tau\left(\otimes\left(\begin{array}{cc}
a & \alpha \\
1 & b c
\end{array}\right)\right)=\tau\left(\otimes\left(\begin{array}{cc}
a & \alpha \\
1 & b
\end{array}\right)\right) c .
$$


If we define $v: B \rightarrow M$ by $v(\alpha)=\tau\left(\otimes\left(\begin{array}{cc}1 & \alpha \\ 1 & 1\end{array}\right)\right)$ then we get:

$$
\tau\left(\otimes\left(\begin{array}{cc}
a & \alpha \\
1 & b
\end{array}\right)\right)=a \tau\left(\otimes\left(\begin{array}{cc}
1 & \alpha \\
1 & 1
\end{array}\right)\right) b=a v(\alpha) b .
$$

We will denote the 2 -cocycle $\tau$ by $\sigma_{v}$. One can easily check that $v(\alpha \beta)=\varepsilon(\alpha) v(\beta)+$ $v(\alpha) \varepsilon(\beta)$ (i.e. $\left.v \in \operatorname{Der}_{k}(B, M)\right)$.

If $\sigma_{v}=\delta_{1}^{\varepsilon}(w)$ for some $w: A \rightarrow M$, then we must have

$$
a v(\alpha) b=a \varepsilon(\alpha) w(b)-w(a \varepsilon(\alpha) b)+w(a) \varepsilon(\alpha) b .
$$

For $\alpha=1$ we get that $w(a b)=a w(b)+w(a) b$ and so $w \in \operatorname{Der}_{k}(A, M)$. If in equation (6) we take $a=b=1$ then we have

$$
v(\alpha)=w(\varepsilon(\alpha))
$$

which concludes our proof.

Next, we want to show that $\Phi_{2}$ need not be one to one. For this let $A=M=k[X]$, $f(X) \in k[X], B=k[f]$, and let $\varepsilon: B \rightarrow A, \varepsilon(f)=f(X)$.

For $q(X) \in k[X]$ we consider $\sigma_{q(X)}: A \otimes A \otimes B \rightarrow A$ defined by

$$
\sigma_{q(X)}(P(X) \otimes Q(X) \otimes \alpha(f(X)))=q(X) P(X) Q(X) \alpha^{\prime}(f(X)) .
$$

One can see that $\delta_{2}^{\varepsilon}\left(\sigma_{q(X)}\right)=0$. Since $H^{2}(A, A)=0$ we have that $H^{2}((A, B, \varepsilon) ; M)=$ $\operatorname{ker}\left(\Phi_{2}\right)$, so every $\widehat{\sigma} \in H^{2}((A, B, \varepsilon) ; M)$ is cobordant to $\sigma(a \otimes b \otimes \alpha)=a v(\alpha) b$, for $v \in \operatorname{Der}_{k}(B, M)$ (here $\widehat{\sigma}$ is the class of $\sigma$ ). With this remark we can prove the following result:

Proposition 6.3. Let $\widehat{\sigma} \in H^{2}((A, B, \varepsilon) ; M)$; then there exists $q(X) \in k[X]$ such that $\widehat{\sigma}=\widehat{\sigma_{q(X)}}$. Moreover, if $p(X), q(X) \in k[X]$ then $\widehat{\sigma_{q(X)}}=\widehat{\sigma_{p(X)}} \in H^{2}((A, B, \varepsilon) ; M)$ if and only if $\widehat{p(X)}=\widehat{q(X)} \in k[X] /\left(f^{\prime}(X)\right)$.

Proof. On $M=k[X]$ we have the $k[f]$-bimodule structure determined by

$$
f \cdot P(X)=f(X) P(X) .
$$

Let $u \in \operatorname{Der}_{k}(B, M)$ and take $q(X)=u(f)$. Then $u(\Lambda(f))=\Lambda^{\prime}(f(X)) q(X)$.

Let $t \in \operatorname{Der}_{k}(A, M)$, and take $t(X)=r(X) \in k[X]$. We have that

$$
t(P(X))=P^{\prime}(X) r(X),
$$

and so $t(\varepsilon(\Lambda(f)))=t(\Lambda(f(X)))=\Lambda^{\prime}(f(X)) f^{\prime}(X) r(X)$. Now the result follows directly from Proposition 6.2.

Remark 6.4. If $f(X) \in k[X]$ has the property that the ideal generated by $f^{\prime}(X)$ is not trivial then the map $\Phi$ is not one to one. Take for example $n \geqslant 2$ and $f(X)=X^{n}$ such that $n$ does not divide the characteristic of $k$. Then we have that

$$
\operatorname{dim}_{k}\left(H^{2}\left(\left(k[X], k\left[X^{n}\right], \varepsilon\right) ; k[X]\right)\right)=n-1 .
$$

Remark 6.5. Using the results from [12], one can notice that the element $\widehat{\sigma_{p(X)}} \in$ $H^{2}((A, B, \varepsilon) ; M)$ corresponds to the $B$-algebra structure on $A[[t]]$ defined by the morphism $\varepsilon_{t}: k[f(X)] \rightarrow k[X][[t]]$ where $\varepsilon_{t}(f(X))=f(X)+t p(X)$. 
More generally, consider $A=M=k[X, Y]$. Let $f(X, Y), g(X, Y) \in A=k[X, Y]$, take $B=k[f, g]$, and define $\varepsilon: k[f, g] \rightarrow k[X, Y]$ determined by $\varepsilon(f)=f(X, Y)$ and $\varepsilon(g)=g(X, Y)$. For any $a(X, Y)$ and $b(X, Y) \in k[X, Y]$ we can define $\sigma_{a, b}: A \otimes A \otimes$ $B \rightarrow A$ by

$$
\begin{gathered}
\sigma_{a, b}(P(X, Y) \otimes Q(X, Y) \otimes \Lambda(f, g))= \\
P(X, Y) Q(X, Y)\left(\frac{\partial \Lambda}{\partial f}(f(X, Y), g(X, Y)) a(X, Y)+\frac{\partial \Lambda}{\partial g}(f(X, Y), g(X, Y)) b(X, Y)\right)
\end{gathered}
$$

for all $P(X, Y), Q(X, Y) \in k[X, Y]$ and $\Lambda(f, g) \in k[f, g]$.

Proposition 6.6. Let $\widehat{\sigma} \in \operatorname{ker}\left(\Phi_{2}: H^{2}((A, B, \varepsilon) ; M) \rightarrow H^{2}(A, M)\right)$, then there exist $a(X, Y), b(X, Y) \in k[X, Y]$ such that $\widehat{\sigma}=\widehat{\sigma_{a, b}} \in H^{2}((A, B, \varepsilon) ; A)$. Moreover, $\widehat{\sigma_{a, b}}=$ $\widehat{\sigma_{c, d}}$ if and only if there exist $v(X, Y)$ and $w(X, Y) \in k[X, Y]$ such that

$$
\left(\begin{array}{c}
a(X, Y)-c(X, Y) \\
b(X, Y)-d(X, Y)
\end{array}\right)=\left(\begin{array}{cc}
\frac{\partial f}{\partial X}(X, Y) & \frac{\partial f}{\partial Y}(X, Y) \\
\frac{\partial g}{\partial X}(X, Y) & \frac{\partial g}{\partial Y}(X, Y)
\end{array}\right)\left(\begin{array}{c}
v(X, Y) \\
w(X, Y)
\end{array}\right) .
$$

Proof. The proof is similar to that of Proposition 6.3. On $M=k[X, Y]$ we have the $k[f, g]$-bimodule structure determined by $f \cdot P(X, Y)=f(X, Y) P(X, Y)$ and $g$. $P(X, Y)=g(X, Y) P(X, Y)$.

Let $u \in \operatorname{Der}_{k}(B, M)$ and take $a(X, Y)=u(f)$ and $b(X, Y)=u(g)$, then

$$
u(\Lambda(f, g))=\frac{\partial \Lambda}{\partial f}(f(X, Y), g(X, Y)) a(X, Y)+\frac{\partial \Lambda}{\partial g}(f(X, Y), g(X, Y)) b(X, Y) .
$$

Let $t \in \operatorname{Der}_{k}(A, M)$, and take $t(X)=v(X, Y)$ and $t(Y)=w(X, Y) \in k[X, Y]$. We have that $t(P(X, Y))=\frac{\partial P}{\partial X}(X, Y) u(X, Y)+\frac{\partial P}{\partial Y}(X, Y) v(X, Y)$ and so

$$
\begin{gathered}
t(\varepsilon(f))=t(f(X, Y))=\frac{\partial f}{\partial X}(X, Y) v(X, Y)+\frac{\partial f}{\partial Y}(X, Y) w(X, Y), \\
t(\varepsilon(g))=t(g(X, Y))=\frac{\partial g}{\partial X}(X, Y) v(X, Y)+\frac{\partial g}{\partial Y}(X, Y) w(X, Y) .
\end{gathered}
$$

Now the result follows directly from Proposition 6.2.

Remark 6.7. A similar statement can be proved if we take $A=k\left[X_{1}, \ldots, X_{n}\right], B=$ $k\left[f_{1}, \ldots, f_{n}\right]$ and $\varepsilon\left(f_{i}\right)=f_{i}\left(X_{1}, \ldots, X_{n}\right) \in k\left[X_{1}, \ldots, X_{n}\right]$.

Remark 6.8. From Proposition 6.6 we know that $\operatorname{ker}\left(\Phi_{2}\right)$ is isomorphic with the space $(k[X, Y] \oplus k[X, Y]) / \operatorname{Image}(J(f, g))$, where

$$
J(f, g): k[X, Y] \oplus k[X, Y] \rightarrow k[X, Y] \oplus k[X, Y]
$$

is determined by the Jacobian matrix associated to the pair $(f(X, Y), g(X, Y))$.

When $k$ is a field with $\operatorname{char}(k)=p, f(X, Y)=X+X^{p}$ and $g(X, Y)=Y+Y^{p}$ then one can see that Image $(J(f, g))=k[X, Y] \oplus k[X, Y]$ and $\varepsilon$ is not onto. It is possible to have $\operatorname{ker}(\Phi)=0$ without the map $\varepsilon$ being surjective. However, when $\operatorname{char}(k)=0$ we can give the following reformulation, for polynomials in two variables, of the Jacobian problem stated in [13] ( $n$ variables if we consider Remark 6.7).

Conjecture 6.9. Let $k$ be a field, char $(k)=0$. Take $A=k[X, Y], B=k[f, g], \varepsilon(f)=$ $f(X, Y)$, and $\varepsilon(g)=g(X, Y)$. If $\Phi_{2}: H^{2}((A, B, \varepsilon) ; A) \rightarrow H^{2}(A, A)$ is one to one, then $\varepsilon$ is surjective. 
Remark 6.10. Notice that from Proposition 6.2 we have an exact sequence:

$$
H^{1}(A, M) \stackrel{\varepsilon^{*}}{\rightarrow} H^{1}(B, M) \stackrel{\chi}{\rightarrow} H^{2}((A, B, \varepsilon) ; M) \stackrel{\Phi_{2}}{\rightarrow} H^{2}(A, M) .
$$

It is reasonable to believe that this can be extended to a long exact sequence. Also, one can ask if the secondary cohomology can be seen as a derived functor (Ext functor) in an appropriate category. We are planning to investigate these problems in a follow-up paper.

\section{Acknowledgment}

We thank the anonymous referee for his/her recommendations that helped us improve this paper. We are especially grateful for suggesting the operad approach of Section 3, which allowed us to improve our initial result.

\section{References}

[1] M. Aguiar and M. Livernet, The Associative Operad and the Weak Order on the Symmetric Groups, Journal of Homotopy and Related Structures 2 (2007), 57-84.

[2] M. Barr, Harrison Homology, Hochschild Homology and Triples, Journal of Algebra 8 (1968), 314-323.

[3] M. Gerstenhaber, The Cohomology Structure of an Associative Ring, Ann. of Math. (2) 78 (1963), 267-288.

[4] M. Gerstenhaber, On the Deformation of Rings and Algebras, Ann. of Math. (2) 79 (1964), 57-103.

[5] M. Gerstenhaber and S.D. Schack, Algebraic Cohomology and Deformation Theory, Kluwer Acad. Publ., Dordrecht, NATO Adv. Sci. Inst. Ser. C Math. Phys. Sci. 247 (1988), 11-264.

[6] M. Gerstenhaber and S.D. Schack, A Hodge-type Decomposition for Commutative Algebra Cohomology, Journal of Pure and Applied Algebra 48 (1987), 229-247.

[7] M. Gerstenhaber and A.A. Voronov, Homotopy G-algebras and Moduli Space Operad, International Mathematics Research Notice 3 (1995), 141-153.

[8] G. Hochschild, On the Cohomology Groups of an Associative Algebra, Ann. of Math. (2) 46 (1945), 58-67.

[9] J.L. Loday, Cyclic Homology, Springer-Verlag, Grundlehren der mathematischen Wissenschaften 301 (1992).

[10] M. Markl, S. Shnider and J. Stasheff, Operads in Algebra, Topology and Physics, Mathematical Surveys and Monographs 96 (2002).

[11] M.D. Staic, Secondary Cohomology and k-invariants, B. Belg. Math. Soc. 19 (2012), 561-572. 
[12] M.D. Staic, Secondary Hochschild Cohomology, 2013, arXiv:1311.7124.

[13] S. Wang, A Jacobian Criterion for Separability, J. of Algebra 65 (1980), 453494.

Mihai D. Staic mstaic@bgsu.edu

Department of Mathematics and Statistics, Bowling Green State University, Bowling Green, OH 43403 USA

Institute of Mathematics of the Romanian Academy, P.O. Box 1-764, RO-70700 Bucharest, Romania.

Alin Stancu stancu_alin1@columbusstate.edu

Department of Mathematics, Columbus State University, Columbus, GA 31907 USA 